\title{
HISTORIA Y PERSPECTIVAS ACERCA DE LA EDUCACIÓN TÉCNICA DE NIVEL SUPERIOR
}

\section{Introducción}

La enseñanza, en todas las épocas y en todo grupo social, se refiere al traspaso de cocimientos, técnicas, costumbres y comportamientos que los adultos consideran necesarios para que sus hijos puedan subsistir, desarrollarse e insertarse de manera plena en la sociedad que los cobija.

En la antigüedad, tanto en Europa como en América, la enseñanza no fue la misma para niños y niñas, del mismo modo que tampoco lo fue para niños y niñas de diferentes grupos sociales. A pesar de las muchas heterogeneidades, tanto entre las diferentes sociedades como al interior de éstas, un hecho ha sido permanente desde los principios de los sistemas formales de enseñanza: siempre se ha ofrecido una educación de corte humanista y científica, y, paralelamente, una vertiente que inicialmente se denominó "vocacional" y que hoy día se designa como "técnica" y "profesional".

La enseñanza técnica es una modalidad estrechamente vinculada con el espacio laboral, antes ligada a la elaboración de productos específicos y hoy a productos y servicios. Por ello, en lo que se refiere a los contenidos de su enseñanza, está unida y supeditada a los requerimientos del mundo del trabajo. Ha cambiado porque los conocimientos y las destrezas necesarias para desempeñar trabajos técnicos también cambian, y ostensiblemente. En Chile la enseñanza técnica era parte de la educación primaria; hoy se imparte mayoritariamente en la enseñanza secundaria y, cada vez más, en la superior.

Su estructura en el país se ha ido construyendo a través de disposiciones legales y recursos económicos, aportados tanto por el 
Estado como por los privados. La intención de crear establecimientos y estructurar el sistema para beneficio de la población se vio frustrada muchas veces: en el siglo XIX a consecuencia de las guerras, y durante el siglo XX por convulsiones sociales que, en su trasfondo, denotan maneras radicalmente opuestas de organización social. En esos escenarios, la enseñanza técnica no ha recibido un trato prioritario en los hechos, si bien en los discursos ha recibido un respaldo fervoroso.

A medida que el mundo laboral cambia, la enseñanza técnica está obligada a estar en continua sintonía con las nuevas tecnologías y con muchos aspectos que antes no se incluían en sus currículos de enseñanza, por ejemplo, habilidades de administración de recursos (como el tiempo, el dinero y los insumos para la producción), la organización del trabajo de grupos de personas, conocimientos de atención al cliente y de computación, y dominio de la expresión oral y escrita.

La enseñanza técnica de nivel superior no es la que concentra actualmente los mayores índices de matrícula, como ha sido tradicional en nuestra historia; sin embargo, a medida que sus egresados demuestren su aporte al mundo laboral, esta circunstancia puede cambiar.

\section{Breve historia de la enseñanza técnica en Chile}

Desde hace mucho tiempo, en los sistemas formales de enseñanza, se ha ofrecido una modalidad orientada a la filosofía, las ciencias y las humanidades, y otra dirigida al dominio de técnicas y tecnologías de producción de bienes y servicios, quedando las artes vinculadas con ambas.

En Europa, el origen de la enseñanza técnica se asocia con las actividades de los gremios de artesanos que se constituyeron en las ciudades feudales. Ellos traspasaron técnicas y conocimientos de cada uno de sus oficios a los aprendices, al tiempo que se organizaron para 
la producción y venta de sus productos. Los aprendices eran, muchas veces, los hijos de los maestros y recibían su formación en un proceso que duraba varios años. Con frecuencia, la actividad dio origen al apellido de las familias; por ejemplo, en alemán "Becker" significa panadero, "Smith" es herrero en inglés y así muchos otros.

En España, los acuerdos del Rey con los gremios de artesanos hicieron posible la introducción de esta modalidad en las Colonias de América, cuando llegaron maestros con conocimientos en diferentes oficios. No obstante, los registros de la enseñanza de oficios durante ese período en Chile se encuentran en la historia de las órdenes religiosas. En el ámbito de la enseñanza tradicional y técnica en Chile tuvieron particular relevancia los Dominicos, Jesuitas y Salesianos.

En 1748, los Jesuitas fundaron una escuela industrial en Calera de Tango, la más grande de la Colonia en Chile. Tal como señala el padre W. Hanisch E. sj (1974), esa casa ha sido denominada "la cuna de la industria chilena": "la casa tenía siete patios (...) en el primero estaba la herrería (...), el segundo servía de vivienda de los padres y se encontraba la relojería y platería, en el tercero había dos alambiques y una rueda grande y una canaleta para el movimiento de la rueda para regar por más de una cuadra a la izquierda (...) en el cuarto, la fábrica de paños u obraje, y elementos para el teñido". Para realizar estos trabajos, trajeron desde Alemania hermanos coadjutores. Ellos eran maestros arquitectos, escultores, plateros, torneros, ebanistas, tejedores, sastres, herreros, boticarios, pintores y alfareros. En una chacra al oriente de Santiago se instaló una fábrica de cerámica, y el lugar pasó a llamarse "la ollería", por la gran cantidad de loza que allí se fabricaba. En todos estos lugares hubo esclavos, jóvenes indígenas y mestizos como aprendices; de esa forma, propagaron por el territorio nacional la enseñanza de sus oficios.

Respecto de los funcionarios de la Corona, el precursor de la enseñanza vocacional en Chile durante el siglo XVIII, a juicio de Amanda Labarca (1939), fue Manuel de Salas. Dado que le tocó ocupar cargos de administración de obras en Santiago, se percató 
de la dificultad para encontrar personas calificadas en trabajos prácticos; de allí nació su interés por ofrecer un tipo de enseñanza que impulsara la actividad minera, agrícola y de industrias: "solamente con el ejercicio y la práctica de conocimientos útiles se acostumbra el espíritu a la exactitud y al raciocinio. De este modo se purgan los ánimos del escolasticismo y espíritu de partido que, después de trastornar el juicio, inspiran una terquedad que trasciende a la sociedad y costumbres, que siempre se resienten de aquella futilidad y orgullo consiguientes a los estudios de memoria, muy diversos de la sinceridad y modestia inseparables de los que sólo estudian la verdad, que se habitúan a ella a fuerza de buscarla y que fundan sus más sublimes discursos en principios sencillos y ciertos".

Por aquel tiempo, la enseñanza de niños y niñas, en lo que se refiere a contenido, era muy distinta. A las niñas se les enseñaba religión, danza, dibujo canto y, en lo posible, a tocar un instrumento musical; a veces se las excluía de la enseñanza de la lectura y escritura. Todos los primeros esfuerzos públicos por extender la enseñanza de los oficios se dirigen sólo a niños y jóvenes varones.

Durante los primeros años de la República se observa el primer interés por la enseñanza técnica en el sistema formal de educación. En 1843, el entonces ministro de Educación, Manuel Montt, apoyó la idea de una escuela técnica, que más adelante se denominaría de Artes y Oficios. Fernando Campos H. (1960) recuerda que uno de los argumentos del ministro fue que "no basta desarrollar la inteligencia de los ciudadanos en las escuelas primarias; es necesario también aumentar sus medios y bienestar".

Las intenciones de crear espacios de enseñanza vocacional se encuentran en los discursos de la época, pero, en los hechos, la Escuela de Artes y Oficios se concretó recién en 1849, en una casa que ocupaba más de media manzana en el sector poniente de Santiago. Diez años antes, la Sociedad Nacional de Agricultura le había insinuado al gobierno la necesidad de implantar en Chile una enseñanza de tipo industrial. La iniciativa era apoyada por el rector 
del Instituto Nacional, Antonio Varas y, de manera especial, por el ministro de Instrucción Pública y luego presidente de la República, Manuel Montt.

Luego de los trámites de rigor, la Escuela de Artes y Oficios se inauguró un 18 de septiembre y contó con un curso de 22 estudiantes, doce de los cuales eran de Santiago. Los cursos duraban cuatro años y comprendían enseñanza teórica y práctica. Entre las primeras, Gramática, Historia y Geografía de Chile, Música y Matemáticas. El dibujo, materia de gran importancia, se dividía en ornamental, de máquinas, lineal, geometría descriptiva y croquis de máquina. Ya en 1855 los talleres incluían Mecánica, Herrería, Fundición, Carpintería, Caldería, Ebanistería, Carretería y Hojalatería.

Gonzalo Vial (1981) afirma que "en 1854 el Fisco mantenía una enseñanza técnica representada sólo por la escuela de Artes y Oficios, dos o tres rudimentarios y oscuros institutos agrícolas y algunas modestas instituciones que cultivaban el dibujo, la pintura, la música, el relieve ornamental, etc. El año 1902 debía agregarse a la escuela citada, por lo menos el Instituto Agrícola, cuatro escuelas prácticas en esa misma área general (Santiago, Chillán, Concepción y Chiloé), una dedicada a la viticultura, (Cauquenes), otra de pesca (Valparaíso) y tres mineras (Copiapó, La Serena y Santiago), la Escuela de Bellas Artes, el Conservatorio Nacional de Música, la Escuela de Ciegos, el Instituto de Sordomudos, el santiaguino instituto Técnico-Comercial (que adquiriría diez congéneres al quinquenio siguiente) y nueve planteles de enseñanza profesional para niñas (serían 29 cinco años más tarde). La matrícula conjunta que reunían los establecimientos nombrados el año 1902, con ser todavía muy poco significativa, debía superar, no obstante, los dos mil educandos".

Respecto de la enseñanza de mujeres, de acuerdo con el profesor Martín Pino (2003), "en 1877 se abrió en Santiago la Escuela Taller para Niñas, ofreciendo enseñanza en Bordado y Moda, y en 1888 la Escuela Industrial de Mujeres, que entregaba conocimientos en Comercio y Moda. Las estudiantes ingresaban con 12 años 
cumplidos, debían ser recomendadas por buena conducta y demostrar conocimientos obtenidos en la enseñanza primaria". Con el paso de los años, las escuelas profesionales para niñas se expandieron a lo largo del territorio nacional y en 1907 ya funcionaban 29 de ellas en las más grandes ciudades, con una cantidad de alumnas no superior a 2.400 jóvenes.

Durante la segunda mitad del siglo XIX tuvo lugar una pugna entre quienes respaldaban la enseñanza técnica y quienes no. Valentín Letelier, citado por Gonzalo Vial (1981), expresaba que "toda instrucción especial, porque va tras el lucro, propende de suyo a materializar el alma. Es lo que todo corazón puro nota al contacto con aquellos hombres que en hora temprana de su vida se someten a sistemas de educación comercial e industrial. Es lo que resalta y repugna en la cultura general norteamericana. En los espíritus así educados no hay más preocupaciones que la del enriquecimiento, atrofian sus sentimientos más nobles y delicados y no comprenden como el filántropo y el político se avienen a consumir su actividad en obras improductivas".

El mismo Vial (1981) menciona que "algunos años después, Augusto Orrego, ministro de Instrucción Pública bajo el gobierno de Federico Errázuriz Echaurren, propiciaba un instituto técnico comercial (...) el primero que hubiésemos tenido. Suscitó instantáneamente violentas críticas en el Senado, el Ministerio y la Universidad. Los senadores le reprochaban quisiese organizar el instituto mediante simple decreto, correspondiendo ello a una ley. Los burócratas educacionales, subordinados al propio Orrego, (lo calificaban como) una cosa más o menos inútil. Y la universidad, con algunos de los míos a la cabeza -según Martina Barros, la mujer del maltratado ministro-, decían que Augusto, al crear el Instituto Técnico Comercial, iba a fomentar la flojera (...), estudios sin importancia, sin la seriedad que da el bachillerato".

A principios del siglo XX, Francisco Antonio Encina (1986) afirmó que era necesario reformar el sistema de enseñanza hacia uno 
más orientado a las actividades productivas. No solamente señaló las deficiencias de la educación sino que también la hacía responsable de la inferioridad económica del país.

Durante la primavera de 1919, Federico Santa María le señaló a su albacea Agustín Edwards "que Chile sufría de tal exceso de profesionales liberales que eran frecuentes y numerosos los casos de abogados, médicos e ingenieros que abandonaban el ejercicio de su profesión para dedicar sus actividades a ramos de índole totalmente diversa. Y que la tendencia era, en el siglo XX, al desarrollo mecánico del mundo y a la generación del trabajo manual, y en Chile, país que según él tenía un territorio singularmente adecuado para desarrollar industrias y una raza con mentalidad y intuición mecánica, era indispensable crear un gran centro educativo que le abriese a las futuras generaciones estos nuevos horizontes y las habilidades para afrontar la lucha por la vida en el campo de la actividad industrial. Le habló al autor con singular entusiasmo de la necesidad de educar a las nuevas generaciones, no imbuyéndolas de conocimientos teóricos y de ciencias abstractas, sino enseñándole prácticamente a fabricar objetos y servirse de sus manos con igual eficacia que de sus cerebros".

Durante esta época Chile tenía una población de poco más de tres millones de personas, de las cuales casi un 60\% vivía en el campo. Hacia "1907 (...) el 42\% de los hombres sabía leer y el 37\% de las mujeres; en el área rural era capaz de leer el 29\% de los habitantes; y en la urbana el 54\%" (Zemelmann, 2004).

En 1920 entró en vigencia la nueva Ley de Educación Primaria que hizo obligatoria la enseñanza, asegurada por el Estado y las municipalidades, para niños y niñas entre los siete y los 13 años de edad. Al mismo tiempo, las escuelas se dividieron en elementales, superiores y vocacionales. En estas últimas se enseñaría industrias manuales, comercio u otros ramos prácticos, minería y agricultura.

Desde la Dirección General del ministerio, Luis Galdames, en 1930, propuso infructuosamente unir los dos tipos de enseñanza, la 
técnica y la general, para que ambas se dieran en los liceos. Al respecto señaló: "y es digno de observarse que algunos que combatieron la reforma decían fundarse en los principios teóricos de Letelier (...) esos principios causaron la desvalorización social de los estudios técnicos y de las profesiones correspondientes (...) toda una generación ha luchado por rectificar ese criterio y ha luchado en vano" (Vial, 1981). Muchos países no separaron radicalmente un tipo de enseñanza de la otra. Es frecuente observar -en Estados Unidos y Australia, por ejemplo- que en los establecimientos de enseñanza secundaria los estudiantes toman algunos cursos prácticos, pero ello no los aleja del currículo de la formación general. En Chile, la enseñanza técnica ofrece en los colegios secundarios un currículo ligado a la especialidad y los estudiantes dejan de estudiar materias de formación general que cada vez se consideran más importantes para los aprendizajes futuros.

Desde la ley de 1920 hasta el comienzo de la segunda guerra mundial se sucedieron en el país varios intentos por mejorar la enseñanza tanto rural como urbana. Planes como el de San Carlos, en 1943, y la Comisión Especial liderada por Irma Salas, en 1945, no se pudieron llevar a efecto por falta de recursos económicos. Por otra parte, los índices de analfabetismo de la población continuaban muy altos. Al mismo tiempo, se expandió la demanda educacional y los establecimientos comenzaron a mostrar graves deterioros.

En este contexto, se creó la Universidad Técnica del Estado, en 1947, institución que nació, en realidad, de la antigua Escuela de Artes y Oficios, que luego se transformó en la Escuela de Ingenieros Industriales y el Instituto Pedagógico Técnico. En lo central, estas modificaciones a la antigua escuela de Artes y Oficios respondieron a la necesidad creciente de más años de preparación en las diversas actividades técnicas. Los estudiantes de la antigua escuela pasaron a obtener formación terciaria como un requerimiento del medio. La Universidad Técnica del Estado fue una continuación de las escuelas de minas en el norte del país y de las agrícolas en todo el territorio nacional ${ }^{1}$.

1 Ver sitio en internet: http://www/usach.cl 
En el ámbito de la educación se produjo un gran cambio en el mundo después de la segunda guerra mundial. La UNESCO expresó que la educación era una inversión en capital humano y los países debían promoverla en todos los niveles, ya que estaba asociada al desarrollo. Esta mirada fortaleció el vínculo entre la educación y los sectores productivos.

En Chile, durante el gobierno de Jorge Alessandri, la Comisión Nacional de Planeamiento sentó las bases de lo que sería la gran reforma educacional del año 1965. Con ésta, se extendió la enseñanza básica a ocho años y se instauró la enseñanza media de cuatro años con las modalidades científico-humanista y técnico-profesional. Esta reforma se caracterizó por una aplicación rápida y por la gran cantidad de profesores que se capacitaron y de escuelas que se construyeron en el territorio nacional. En el ámbito de la educación de adultos -que seguía siendo urgente de atender, por las altas tasas de analfabetismo y la baja escolaridad de la población en general- se promovió la enseñanza técnica de nivel superior. En el marco de esas políticas, complementarias de la gran reforma de la enseñanza secundaria, se creó el Instituto Nacional de Capacitación Profesional, INACAP, el cual, desde CORFO, comenzó a implementar cursos de perfeccionamiento en oficios técnicos a adultos.

Durante la década de los sesenta la creación de INACAP estaba implícita en la idea de la Universidad Laboral: aquella que entregara a los trabajadores un camino hacia la enseñanza superior.

Las convulsiones político-sociales que acompañaron al derrocado gobierno de Salvador Allende impidieron su anunciada reforma educacional. Por ese tiempo se difundió el eslogan "universidad para todos", que en la práctica no se materializó. Sin embargo, se mantuvieron los espacios para la enseñanza técnica de adultos y se impulsaron programas de capacitación para trabajadores en institutos como INACAP y el Departamento Universitario Obrero Campesino de la Universidad Católica (DUOC), al tiempo que se promovió la apertura de carreras técnicas en universidades como 
la Técnica Federico Santa María y Técnica del Estado (de Santiago), fundamentalmente.

Entre 1973 y 1989 se introdujeron reformas significativas en el ámbito de la enseñanza superior en Chile. A diferencia de los años sesenta, que fomentaron un planeamiento centralizado de la actividad económica y de las actividades del Estado en general, Augusto Pinochet, bajo el concepto del Estado subsidiario, introdujo una visión diametralmente opuesta para el desarrollo económico y social del país. En el área de la educación se dio espacio a personas naturales y/o jurídicas para crear establecimientos educacionales en todos los niveles. Tras la excesiva politización de la sociedad chilena después de los gobiernos de Eduardo Frei Montalva y Salvador Allende, "donde el poder político copó todas la instancias sociales de la vida nacional, como las universidades, municipios y sindicatos" (Moncada, 2006), se planteó que el Estado favorecería a los cuerpos medios de la sociedad para que desarrollaran, con autonomía, la obtención de sus fines específicos. Esta visión se aplicó en el ámbito de administración educacional en todos los niveles. Se traspasó la administración de liceos y escuelas a los municipios y la de muchos liceos técnicos a las agrupaciones empresariales, de acuerdo con sus áreas de especialidad. En el ámbito de la educación técnica superior, la facultad de abrir centros de formación técnica (CFT), mediante el DFL No 24/1981, del Ministerio de Educación, permitió a personas naturales y/o jurídicas presentar a esa secretaría de Estado un instrumento legal constitutivo. Una vez aprobado, se debía acreditar un proyecto de desarrollo institucional que incluyera los recursos docentes, didácticos, económicos y físicos para ofrecer la formación conducente a un título técnico de nivel superior. Ello introdujo la gran expansión de la enseñanza técnica de nivel superior en Chile durante la década de los años ochenta y hasta hoy.

Durante el gobierno de Patricio Aylwin los aspectos relativos a la legislación para crear centros de formación técnica no cambiaron sustantivamente. Desde ese momento, la legislación se ha abocado a crear los mecanismos para asegurar la calidad del servicio que 
ofrecen las instituciones y, últimamente, a abrir nuevos espacios de financiamiento para los estudiantes.

\section{Situación actual}

De acuerdo con los antecedentes del Ministerio de Educación, en abril de 2006 había 395.406 jóvenes matriculados en los 1.382 establecimientos de enseñanza media técnico-profesional en el país. A la misma fecha, en los CFT estaban matriculados 62.589 estudiantes en 100 instituciones.

De lo anterior se desprende que la gran mayoría de los técnicos en Chile se está preparando en la enseñanza media. A ello contribuye el sistema de financiamiento que el Estado otorga actualmente para este tipo de enseñanza. Estos estudiantes reciben el servicio de forma gratuita o casi gratuita; los alumnos de los CFT, en cambio, deben pagar colegiaturas en las diferentes instituciones que, en su mayoría, son privadas y sin acreditación, lo que les impide obtener créditos para financiar sus estudios.

En 1997, el gobierno de la época creó el proyecto MECESUP para mejorar la equidad, promover el desarrollo regional, la investigación, entre otros factores. En el marco de este programa, varios CFT se han visto favorecidos para crear carreras, perfeccionar profesores y adquirir equipamiento; no obstante, hasta la fecha, en ninguno de los concursos los CFT han obtenido más del 10\% de los recursos. En cambio, el programa ha apoyado la creación de nuevos CFT en las universidades del Consejo de Rectores o tradicionales.

Desde 2006 los estudiantes de la enseñanza técnica superior acreditada pueden acceder a un crédito para sus estudios. La Ley No 20.027 abre por primera vez la posibilidad de respaldo a este universo de jóvenes. Los CFT acreditados actualmente son cinco, y concentran un $60 \%$ de la matrícula del sector.

En el país hoy dan servicio educacional unos 100 CFT. Sin embargo, la mayor parte de la matrícula se concentra en unos 
pocos. Además, como el sistema se ha desarrollado rápidamente y en un espacio de alta competencia, muchos se han fusionado. En este escenario "la evidencia parece indicar que aquellos institutos profesionales y centros de formación técnica que han permanecido aislados han visto decrecer su incidencia en el sistema, han mantenido discretos niveles o han disminuido su matrícula total, lo que afecta seriamente sus posibilidades de desarrollo" (Salazar, 2007).

Los estudiantes de la enseñanza técnica de nivel superior provienen, en su mayoría, de los establecimientos municipales y particulares subvencionados. Muchos de ellos necesitan insertarse prontamente en el mundo del trabajo. Un grupo cada vez más numeroso es el de aquellos que no se visualizan en una carrera tradicional. Sienten que su vocación se encuentra en áreas de especialidad como la cocina, el diseño y la mecánica. Para ellos existe cada vez más espacio en los CFT que permanentemente están ofreciendo programas de estudio en esta línea.

El actual sistema de educación superior en Chile no favorece la movilidad entre las instituciones y tampoco se ha establecido una articulación entre la enseñanza media técnico-profesional y la superior. Este último punto es el que el MINEDUC considera una necesidad y reconoce no haber podido establecerla por la forma en que se ha desarrollado el sistema en nuestro país (Álamos, 2006).

En la línea de mejorar la articulación se estableció el programa "Chile Califica", que promovió el desarrollo de redes para favorecer el intercambio de información entre empresarios y establecimientos de enseñanza técnica de nivel medio y superior. El objetivo era establecer mecanismos de reconocimiento de estudios entre las instituciones y recibir la información de las empresas respecto de las necesidades del mundo laboral. Mucho de ello ha ocurrido; sin embargo, no es posible afirmar que este programa haya resuelto los problemas de articulación entre la enseñanza técnica secundaria y la superior.

Los contenidos de la enseñanza técnica de nivel superior son diseñados por especialistas en estrecha vinculación con el mundo 
del trabajo. Actualmente, los CFT autónomos diseñan sus propios programas de estudio, incorporando estándares de equipamiento para impartir clases y los insumos para la enseñanza. Los cambios tecnológicos obligan a las instituciones a actualizar permanentemente sus contenidos y a realizar continuos programas de perfeccionamiento docente. Por estos motivos, se trata de una modalidad de alto costo.

\section{Perspectivas}

Las perspectivas de desarrollo de la enseñanza técnica se relacionan estrechamente con la legislación en que se enmarque y los recursos que, a través del Estado o por medios privados, se aporten a su desarrollo. Durante la década de los noventa, si bien se incrementaron los recursos fiscales y los aportes del sector privado fueron también significativos, se incrementó grandemente la matrícula en la enseñanza superior. No obstante, ello ocurrió en mayor medida en las carreras universitarias. Aun cuando el acceso a la educación terciaria en Chile ha crecido sostenidamente hasta alcanzar un 37\% de la población entre 18 y 24 años, "los recursos (fiscales) se han concentrado en los alumnos de las universidades tradicionales, relegando a un segundo plano a más de la mitad de los alumnos de educación superior, que son los que estudian en universidades privadas, institutos profesionales y centros de formación técnica. El sistema de garantía compartida entre el Estado y las instituciones de educación superior propuesto, en cuanto se orienta a aumentar el flujo de recursos del mercado de capitales a toda la educación superior autónoma y acreditada, tradicional o privada, universitaria o no universitaria, es un reconocimiento de que sin la acción concertada del Estado y el sector privado el financiamiento estudiantil para todos los que lo necesitan es una quimera" (Bernasconi, 2004).

Aunque algunos piensan que "la nueva ley de financiamiento (Ley N 20.027 de 2006) a la educación superior tiene la intención de corregir una situación de inequidad histórica, estimamos que no va a lograr revertir la situación de promover el ingreso al estudio de las carreras técnicas. Ello porque los requisitos que van a poner las 
instituciones para otorgar los préstamos limitarán el acceso masivo de estudiantes. La única manera que efectivamente se pueda generar un sistema de amplia cobertura es que el riesgo de deserción lo absorba el Estado. Si el Estado se resolviera por avalar el riesgo de deserción ello aún no sería suficiente para resolver este desafío, ya que las instituciones financieras no cuentan con la información necesaria para la correcta evaluación de la solicitud de crédito y los mecanismos para conseguir su pago" (Aedo, 2003).

Observando estas perspectivas, cabe preguntarse si es conveniente para el país el gran número de estudiantes que actualmente se forma técnicamente en enseñanza media. Probablemente, este será el aspecto que requiera mayor estudio en los próximos años. Una formación general sólida en lenguaje y matemáticas es cada vez más demandada en el mundo laboral para ejercer actividades técnicas: ¿hasta qué punto es conveniente privar de esa formación general a tantos estudiantes de enseñanza media que actualmente cursan la opción técnico profesional?

Respecto de la inserción laboral, lo que se necesita son personas en condiciones de continuar aprendiendo y adaptándose a los cambios tecnológicos. En lo curricular, una innovación por competencias en el área de las relaciones humanas y la administración general de insumos y personas: saber planificar, administrar el tiempo, organizar a grupos de personas para la producción y obtener resultados satisfactorios serán los requisitos mínimos para el desempeño de especialidades técnicas. Junto con ello, los conocimientos sólidos en el uso de las tecnologías de la información y, en algún grado, el dominio del idioma inglés.

Es probable que la enseñanza técnica de nivel superior atienda cada vez a más estudiantes. Ello si el entorno de apoyo en recursos continúa en los niveles en que se ha dado últimamente.

También es posible prever que se harán más esfuerzos por articular la enseñanza media con el nivel superior, tanto técnico como 
profesional y universitario. La oportunidad de continuar estudiando debería ser expedita para todos quienes puedan y deseen hacerlo.

El historiador Gonzalo Vial ha calificado a la enseñanza técnica en nuestro país como "Cenicienta", y quizás tiene razón. Parece crónico el hecho de que en los discursos y en las palabras los líderes, tanto políticos como educacionales, invocan la importancia de la enseñanza técnica y, sin embargo, no existe en los hechos un respaldo económico y administrativo para elevarla ante los ojos de la ciudadanía.

Tal como Cenicienta, la enseñanza técnica puede ser una reina el día en que sus egresados aporten de tal manera en el mundo laboral que éste reconozca su contribución por medio de mejores remuneraciones. Hay actualmente una brecha entre las remuneraciones de los técnicos y los profesionales universitarios (Larrañaga, 2006). Si la preparación técnica comienza a elevar el nivel de los salarios de sus titulados o, bien, sus estudios contribuyen a que inicien trabajos independientes, va a acaparar las miradas de los jóvenes y de sus familias. Esta modalidad de enseñaza ya favorece la movilidad social, pero si logra cada vez mejores resultados, éstos se reflejarán en una mayor matrícula.

\section{Referencias bibliográficas}

Álamos, Pilar. (2006) Los desafíos de la formación técnica de nivel superior en Chile. Santiago de Chile: Ministerio de Educación, Minuta de Trabajo.

Aedo, Cristián y Dittborn, Paulina. (2003) Oferta y demanda y políticas públicas en la educación superior. Calidad en la Educación, 22, pp. 55-68.

Bernasconi, Andrés. (2006) Donde no somos tigres: Problemas de la formación técnica en Chile y en el contexto latinoamericano. Sitio en Internet. Disponible en www.expansiva.cl

Bernasconi, Andrés y Rojas, Fernando. (2003) La educación superior en Chile 1981-2003. Santiago de Chile: Editorial Universitaria, p. 180. 
Campos, Fernando. (1960) Desarrollo educacional 1810-1960. Santiago de Chile: Editorial Andrés Bello, p. 42.

Edwards, Agustín. (1934) Apuntes biográficos de Federico Santa María. Valparaíso: Impr. Universo, pp.141-142.

Encina, Francisco Antonio. (1986) 1874-1965. Nuestra inferioridad económica, Santiago de Chile: Editorial Universitaria, p. 62.

Hanisch E., Walter sj (1974) Historia de la Compañía de Jesús en Chile. Santiago de Chile: Editorial Francisco de Aguirre S.A., p. 112.

Labarca, Amanda. (1939) Historia de la enseñanza en Chile. Santiago de Chile: Editorial Universitaria, pp. 54-56.

Larrañaga, Osvaldo. (2006) Costos y beneficios de estudiar en centros de formación técnica. ¿Neutralidad de Política Pública? Sitio en Internet. Disponible en www.expansiva.cl

Moncada, Belén. (2006) Jaime Guzmán. Una democracia contrarrevolucionaria El político de 1964 a 1980. Santiago de Chile: Ril Editores, p. 81.

Pino, Martín. (2003) Nuestra cultura tecnológica. Santiago de Chile: Editorial Universidad de Santiago, pp. 101 y 70.

Salazar, José Miguel. (2007) La educación superior no universitaria. Santiago de Chile: Consejo Superior de Educación, comunicación personal.

Vial, Gonzalo. (1981) Historia de Chile (1891-1973) Tomo 1. Santiago de Chile: Editorial Zig-Zag, pp. 66- 169.

Zemelman Myriam; Ayala, Estela; Hermosilla, Patricia, et al. (2004) Seis episodios de la educación chilena. 1920-1965 Santiago de Chile: Universidad Finis Terrae, comunicación personal.

Recibido: 31 de agosto de 2007

Aceptado: 29 de octubre de 2007 